\title{
Design of Matching Circuits for Microstrip Triplexers Based on Stepped-Impedance Resonators
}

\author{
Pu-Hua Deng, Ming-Iu Lai, Student Member, IEEE, Shyh-Kang Jeng, Senior Member, IEEE, and
} Chun Hsiung Chen, Fellow, IEEE

\begin{abstract}
New matching circuits for microstrip triplexers are proposed based on half-wavelength tapped-connected (or fed) stepped-impedance resonators. The stepped-impedance resonators play important roles for the matching circuits, either to serve as a through pass at the center frequency of a bandpass filter or to provide a short circuit at the center frequency of another bandpass filter. First, three tapped-connected stepped-impedance resonators together with suitable branch transmission lines are utilized to develop the matching circuits for a microstrip triplexer. The design procedure for these matching circuits is much simpler than that for the conventional triplexer structures due to the use of tapped-connected stepped-impedance resonators. Second, to reduce the number of stepped-impedance resonators and to improve the spurious resonances associated with the proposed matching circuits, modified matching circuits for the triplexer are also proposed. Agreement between measured and simulated results is observed and supports the usefulness of the design procedure.
\end{abstract}

Index Terms-Microstrip, stepped-impedance resonator, triplexer.

\section{INTRODUCTION}

$\mathbf{M}$ UTIPLEXERS are key components of the transceiver for modern wireless or mobile communication systems. Nowadays, planar circuits are widely adopted due to their compact size and low integration cost using the printed circuit technology [1]. In multiservice and multiband communication systems, diplexers and triplexers are needed to possess the capabilities of high compactness, light weight, and high isolation. Basically, a multiplexer is composed of bandpass filters and associated matching circuits, and thus proper designs of high-performance filters and matching circuits are essential in the development of a multiplexer.

Several diplexer [2]-[5], triplexer [6]-[10], and multiplexer [11] structures have been proposed for the multiservice and multiband communication systems. Recently, planar diplexers and triplexers were demonstrated by properly locating the attenuation poles near the passband of the diplexer and triplexer [8], [9]. In [10], the low-temperature co-fired ceramic (LTCC) diplexer and triplexer were implemented using a parallel-coupled line filter connected with a capacitor. In general, the designs of $\mathrm{T}$-junction and branch lines are essential in the development of diplexers and triplexers because they may affect the characteristic of each filter. In designing the T-junction and

Manuscript received March 20, 2006; revised July 1, 2006. This work was supported by the National Science Council of Taiwan, R.O.C., under Grant NSC 94-2752-E-002-001-PAE and Grant NSC 94-2219-E-002-008.

The authors are with the Department of Electrical Engineering and Graduate Institute of Communication Engineering, National Taiwan University, Taipei 106, Taiwan, R.O.C. (e-mail: chchen@ew.ee.ntu.edu.tw).

Digital Object Identifier 10.1109/TMTT.2006.88616 branch lines for a diplexer or triplexer, one of the ports should be matched at its center frequency and the other port(s) should be look opened. Specifically, only one open condition is needed for diplexer design, while two open conditions are required for triplexer design. Consequently, the design procedure for the triplexer structures is more complicated than that for diplexer structures. Until now, no simplified design procedure has been available for the triplexer structures.

In this study, a systematic design procedure for the matching circuits of microstrip triplexers is presented. The proposed matching circuits are constructed based on the stepped-impedance resonators (SIRs), which are tapped-connected to the branch transmission lines. Usually, the SIRs were used in the filter design so as to reduce the circuit size [12]-[14], to push the spurious passband to the higher frequency, and even to suppress the multiple spurious passbands as discussed in [15] and [16]. In this study, the SIRs are tapped-connected and employed mainly for the matching purpose. By properly designing the tapped-connected SIR to resonate at the center frequency $f_{01}$ of the first bandpass filter and to adjust the tapped position of this SIR so as to provide a short circuit at the center frequency $f_{02}$ of the second bandpass filter, for instance, one of the matching circuits may easily be designed accordingly. However, by an introduction of these three SIRs, spurious resonances would be produced when the total length of any two open stubs is of a half-wavelength $(\lambda / 2)$ in length. To improve the spurious resonances associated with the proposed matching circuits and to reduce the number of SIRs, modified matching circuits for the triplexer are also proposed.

\section{TAPPED-FED SIRS}

The basic matching circuit for the proposed triplexer is composed of two transmission-line sections of characteristic impedances $Z_{01}, Z_{02}$ and electrical lengths $\theta_{1}$ and $\theta_{2}$, which are tapped-connected to an SIR as shown in Fig. 1(a). The SIR is also made of transmission-line sections of characteristic impedances $Z_{a}$ and $Z_{b}$ and electrical lengths $\theta_{a}$ and $\theta_{b}$. The equivalent circuit may be represented by Fig. 1(b), in which $Z_{A}$ and $Z_{B}$ are the input impedances looking into either side of the SIR and $Z_{s}$ is the resultant input impedance to the tapped-connected or tapped-fed SIR looking at the feed point, i.e.,

$$
Z_{s}=\frac{Z_{A} Z_{B}}{Z_{A}+Z_{B}}
$$

The SIR is an essential part of triplexer's matching circuits. It should provide an open circuit $\left(Z_{s}=\infty\right)$ or a through pass to the transmission line sections connected to input/output ports 1 and 2 at the center frequency of one passband, and should also provide an impedance zero $\left(Z_{s}=0\right)$ or a short circuit 


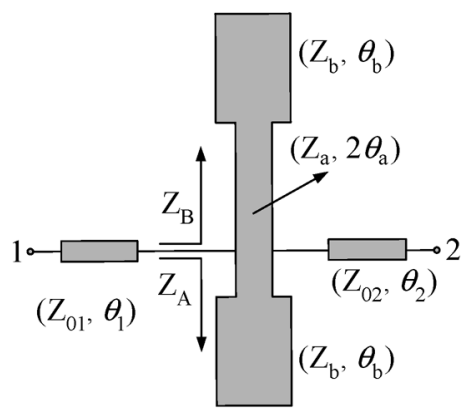

(a)

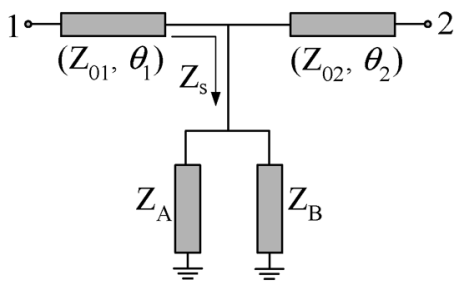

(b)

Fig. 1. (a) Basic matching circuit. (b) Equivalent circuit.

at the two center frequencies of other two passbands. To this end, the tapped-fed SIR structure shown in Fig. 1 needs careful investigation.

The SIR may be tapped connected or fed across the line section of characteristic impedance $Z_{a}$ as shown in Fig. 2(a). From this feed point, the input impedances looking into either side of SIR may be expressed as

$$
\begin{aligned}
Z_{A} & =j Z_{a} \frac{\tan \left(r \theta_{a}\right)-R_{z} \cot \left(\theta_{b}\right)}{1+R_{z} \cot \left(\theta_{b}\right) \tan \left(r \theta_{a}\right)} \\
Z_{B} & =j Z_{a} \frac{\tan \left[(2-r) \theta_{a}\right]-R_{z} \cot \left(\theta_{b}\right)}{1+R_{z} \cot \left(\theta_{b}\right) \tan \left[(2-r) \theta_{a}\right]}
\end{aligned}
$$

where $R_{z}=Z_{b} / Z_{a}$ is the impedance ratio for the SIR and $r$, $0 \leq r \leq 1$, is the variable to specify the feed location. By letting

$$
Z_{A}+Z_{B}=0
$$

one may determine the resonance frequency $f_{r}$ of the SIR structure at which the SIR behaves like an open circuit $\left[Z_{s}\left(f_{r}\right)=\right.$ $\infty]$, and thus it may provide a through pass to the transmission-line sections in Fig. 1. Based on (4), the information about the resonance frequency $f_{r}$ may easily be established and will be used in the design of a triplexer's matching circuits.

By letting $Z_{A}$ or $Z_{B}$ be equal to zero, one may determine the zero frequency $f_{j}$ of the SIR structure at which the SIR behaves like a short circuit $\left[Z_{s}\left(f_{j}\right)=0\right]$. Based on (2) and (3), the equation to determine the zero frequency $f_{j}$ may simply be written as

$$
\tan \left(r \theta_{a}^{\prime}\right)-R_{z} \cot \left(\theta_{b}^{\prime}\right)=0
$$

or

$$
\tan \left[(2-r) \theta_{a}^{\prime}\right]-R_{z} \cot \left(\theta_{b}^{\prime}\right)=0
$$

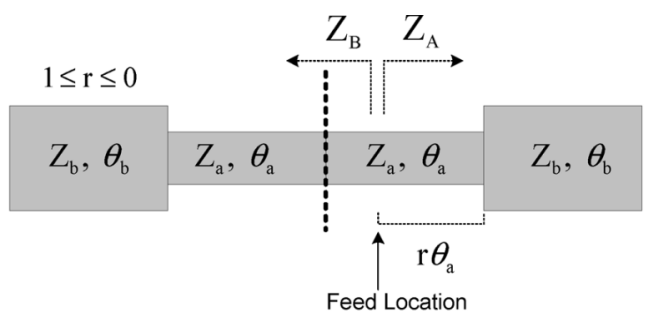

(a)

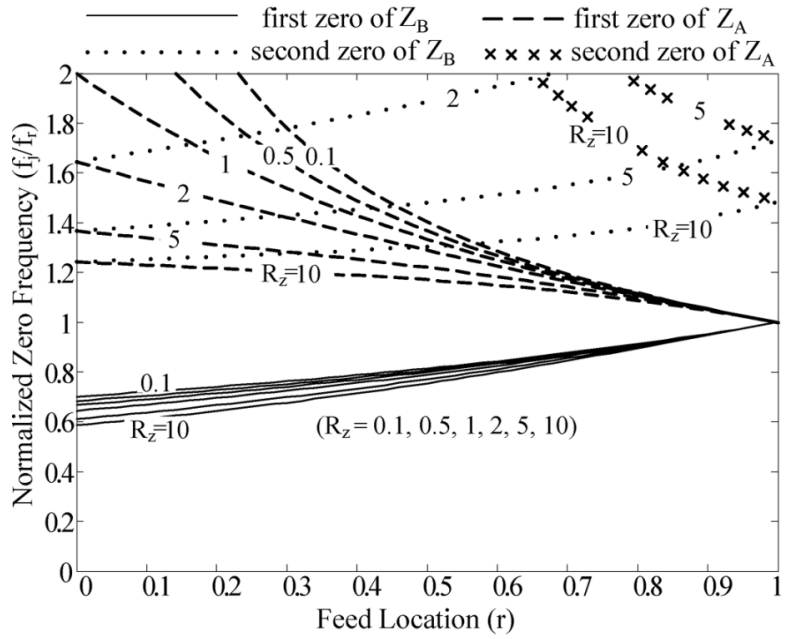

(b)

Fig. 2. (a) SIR tapped fed across the line with impedance $Z_{a}$. (b) Curves to show the relation between the zero frequency $f_{j}$ and the feed location $r$ (normalization with respect to the resonance frequency $f_{r}$ ).

Here, $\theta_{a, b}^{\prime}=\theta_{a, b}^{r}\left(f_{j} / f_{r}\right), f_{r}$ is the resonance frequency of the SIR $\left[Z_{s}\left(f_{r}\right)=\infty\right], f_{j}$ is the zero frequency of the SIR $\left[Z_{s}\left(f_{j}\right)=0\right]$, and $\theta_{a, b}^{r}$ are the electrical lengths of the SIR sections at $f_{r}$. Based on (5) and (6), the impedance zero frequencies may be solved by the root-finding algorithms. For the case of $\theta_{a}^{r}=\theta_{b}^{r}=\tan ^{-1}\left(\sqrt{R_{z}}\right)$ such that $\left[Z_{s}\left(f_{r}\right)=\infty\right]$, the relation between the zero frequency $f_{j}$ and the feed location for the SIR [see Fig. 2(a)] may be depicted in Fig. 2(b), which will be useful in the design of the matching circuits for a triplexer. For the general case of $\tan \left(\theta_{a}^{r}\right) \tan \left(\theta_{b}^{r}\right)=R_{Z}$ [17], which also gives $\left[Z_{s}\left(f_{r}\right)=\infty\right]$, the design curves relating the zero frequency to the feed location may again be obtained in a similar manner. However, the curves for the general case are not included because only the curves for the special case Fig. 2(b) are adopted in the subsequent design of the proposed triplexer.

Alternatively, the SIR may be tapped connected or fed across the line section of characteristic impedance $Z_{b}$, as shown in Fig. 3(a). From this feed point, the impedances looking into either side of the SIR may be written as

$$
Z_{A}=-j Z_{b} \cot \left[(1-r) \theta_{b}\right]
$$

and (8), shown at the bottom of this page. By setting (7) or (8) equal to zero, the impedance zero frequencies associated with

$$
Z_{B}=j Z_{b} \frac{\left[\tan \left(2 \theta_{a}\right)-R_{z} \cot \left(\theta_{b}\right)\right]+\left[1+R_{z} \cot \left(\theta_{b}\right) \tan \left(2 \theta_{a}\right)\right] R_{z} \tan \left(r \theta_{b}\right)}{R_{z}\left[1+R_{z} \cot \left(\theta_{b}\right) \tan \left(2 \theta_{a}\right)\right]-\left[\tan \left(2 \theta_{a}\right)-R_{z} \cot \left(\theta_{b}\right)\right] \tan \left(r \theta_{b}\right)}
$$




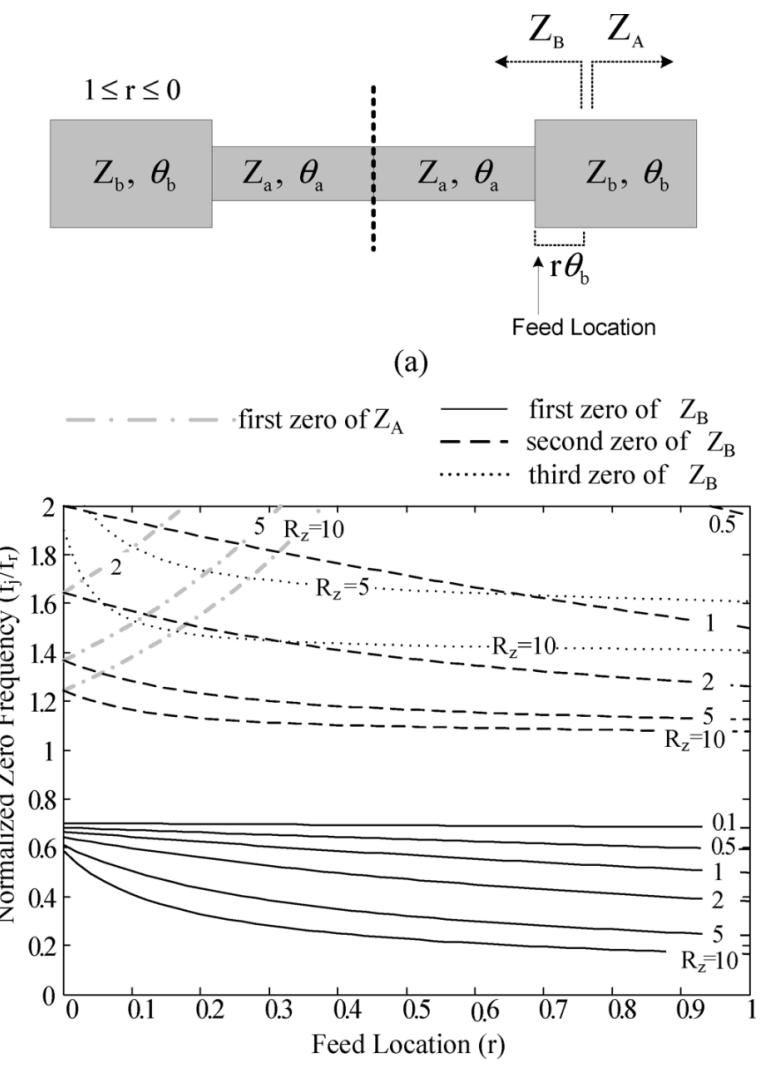

(b)

Fig. 3. (a) SIR tapped fed across the line with impedance $Z_{b}$. (b) Curves to show the relation between the zero frequency $f_{j}$ and the feed location $r$.

the tapped-connected SIR may be determined as follows:

$$
(1-r) \theta_{b}^{\prime}=\frac{2 n+1}{2} \pi, \quad n=0,1,2, \ldots
$$

or

$$
\begin{array}{r}
\tan \left(2 \theta_{a}^{\prime}\right)-R_{z} \cot \left(\theta_{b}^{\prime}\right)+\left[1+R_{z} \cot \left(\theta_{b}^{\prime}\right) \tan \left(2 \theta_{a}^{\prime}\right)\right] \\
\cdot R_{z} \tan \left(r \theta_{b}^{\prime}\right)=0 .
\end{array}
$$

For the particular case of $\theta_{a}^{r}=\theta_{b}^{r}=\tan ^{-1}\left(\sqrt{R_{z}}\right)$, the zero frequencies against the feed location may be depicted in Fig. 3(b). Again, the design curves for the general case, $\tan \left(\theta_{a}^{r}\right) \tan \left(\theta_{b}^{r}\right)=R_{Z}$ [17], may similarly be obtained, but are not included.

\section{MATCHING CIRCUITS FOR TRIPLEXERS}

In general, the design of a triplexer structure is more difficult than that of a diplexer. The design of the branch lines for the triplexer's matching circuits is the key to the triplexer implementation. The design becomes more complicated and usually influences the characteristics of the triplexer.

Fig. 4(a) shows the conventional triplexer structure [9], in which three branch lines are connected to three bandpass filters (BPF1, BPF2, and BPF3). Here, $Z_{\text {ink }}, k=1,2$, or 3 , is the input impedance defined at the left-hand side of each branch line, as shown in Fig. 4(a). The branch line is designed under the conditions of no reflection at the center frequency of one passband and

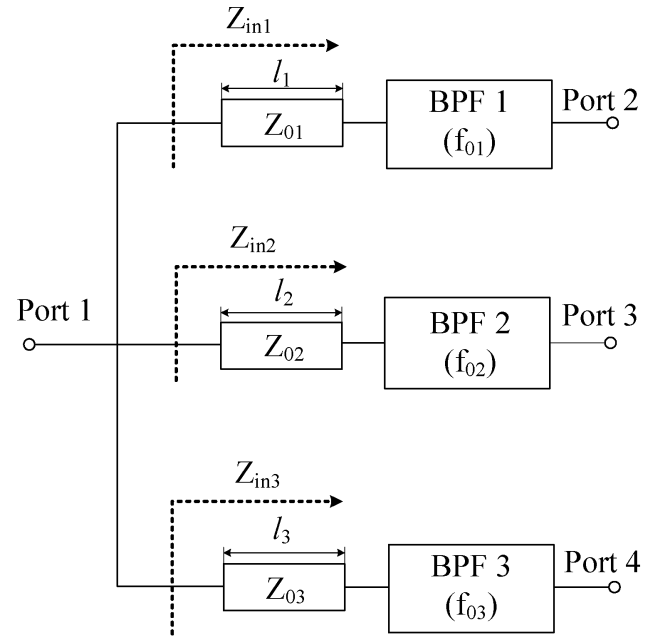

(a)

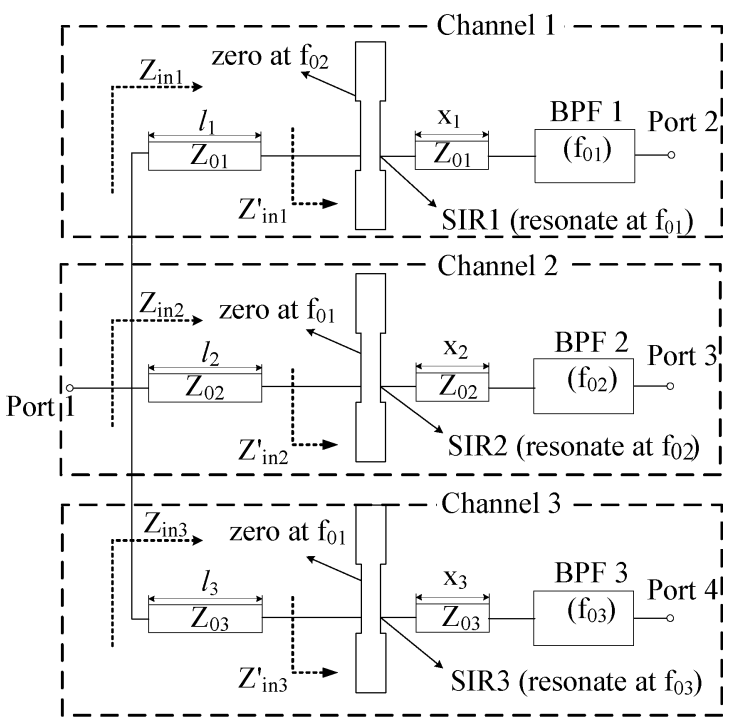

(b)

Fig. 4. Triplexer structures. (a) Conventional. (b) Proposed.

total reflection at the center frequencies of the other two passbands. In [9], the characteristic impedances $Z_{o k}, k=1,2,3$, of the branch lines are set to be $50 \Omega$, and the lengths of branch lines $l_{k}, k=1,2,3$, are chosen such that the following conditions are satisfied:

$$
\begin{aligned}
& Z_{\text {in } 1}\left(f_{01}\right)=50 \Omega \quad Z_{\text {in } 1}\left(f_{02}\right)=Z_{\text {in } 1}\left(f_{03}\right)=\infty \\
& Z_{\text {in } 2}\left(f_{02}\right)=50 \Omega \quad Z_{\text {in } 2}\left(f_{01}\right)=Z_{\text {in } 2}\left(f_{03}\right)=\infty \\
& Z_{\text {in } 3}\left(f_{03}\right)=50 \Omega \quad Z_{\text {in } 3}\left(f_{01}\right)=Z_{\text {in } 3}\left(f_{02}\right)=\infty \text {. }
\end{aligned}
$$

For instance, in designing the first branch line, its length $l_{1}$ should be chosen such that both open conditions in (11) must be fulfilled simultaneously. The step of adjusting one $l_{1}$ to meet two open conditions (11) is rather difficult and usually needs some optimization.

Note that the conventional diplexer does not face with the design difficulty because each branch line is adjusted to meet only one open condition. Consequently, the design of conventional 
triplexer structures is more complicated than that of the diplexer structures.

In this study, simplified matching circuits for the microstrip triplexer composed of three tapped-connected (or tapped-fed) SIRs are proposed. Fig. 4(b) shows the architecture of the proposed triplexer structure. The SIRs play two important roles for the matching circuits. The first one is to provide an impedance zero or a short circuit to the branch line, as mentioned in Section II. By adding a quarter-wavelength $(\lambda / 4)$ transmission line to this short circuit, an open circuit can be obtained to fulfill the open conditions in (11)-(13). The other role is to serve as a through path when the specific SIR resonates at the center frequency of the filter connected to each branch line. Note that the short-circuit property provided by the SIR is quite narrow in bandwidth. The practical bandwidth realized by the use of the SIR is around the order of $10 \%$. For designing a triplexer with the bandwidth exceeding this limit, the passband performance of triplexer may be degraded.

Three SIRs are tapped-connected to suitable branch transmission lines to form the matching circuits for the proposed microstrip triplexer. The branch lines have the characteristic impedances $Z_{0 k}, k=1,2,3$, which are preset to $50 \Omega$, and their lengths are $\left(l_{1}, x_{1}\right),\left(l_{2}, x_{2}\right)$, and $\left(l_{3}, x_{3}\right)$, respectively.

First, the resonator SIR1, for instance, is designed to resonate at $f_{01}$ so as to provide a through path to the filter BPF1 at $f_{01}$, and thereby the condition $Z_{\text {in1 }}\left(f_{01}\right)=50 \Omega$ may be fulfilled. Due to the tapped-connected feature of this resonator, this SIR1 produces several impedance zeros to provide a short circuit to the branch line which is tapped-connected to the SIR1. These impedance zeros are specified by (5), (6), (9), and (10) and are represented in Figs. 2(b) and 3(b).

Second, one of these impedance zeros is selected to locate at the center frequency $f_{02}$ of BPF2 so that $Z_{\mathrm{in} 1}^{\prime}\left(f_{02}\right)=0$. The length of branch line is adjusted to give $l_{1}=\lambda / 4$ at $f_{02}$, so that the condition $Z_{\mathrm{in} 1}\left(f_{02}\right)=\infty$ is satisfied.

Third, the length $x_{1}$ of the branch line is adjusted so that the remaining open condition $Z_{\text {in } 1}\left(f_{03}\right)=\infty$ is met. Note that the adjustment of $x_{1}$ has no influence on the condition $Z_{\text {in } 1}^{\prime}\left(f_{02}\right)=$ 0 . Consequently, the open condition $Z_{\text {in } 1}\left(f_{02}\right)=\infty$ is not altered under the process of adjusting $x_{1}$.

Similarly, the matching circuits for other two channels may easily be designed to meet the open conditions in (12) and (13).

In this study, all of the circuits are fabricated on the Rogers RO4003C substrate $\left(\varepsilon_{r}=3.38, \tan \delta=0.002\right.$, and thickness $h=0.508 \mathrm{~mm}$ ). Fig. 5 shows the layout of the implemented microstrip triplexer, based on the proposed configuration in Fig. 4(b), which has three fourth-order cross-coupled bandpass filters as discussed in [18]. The dimensions of each part in Fig. 5 are given in Table I for further reference. The implemented triplexer has a dimension of $40.45 \mathrm{~mm} \times 120.43 \mathrm{~mm}$. The three matching circuits are realized by the three microstrip SIRs, three $\lambda / 4$ microstrip lines, and another three microstrip lines of proper lengths so as to meet all impedance conditions in (11)-(13).

The measured and simulated results of the implemented triplexer (Fig. 5) are shown in Figs. 6 and 7. For the first passband, the measured center frequency $f_{01}$ is at $1.47 \mathrm{GHz}$, the minimum insertion loss is $3.4 \mathrm{~dB}$, and the $3-\mathrm{dB}$ bandwidth is $4.96 \%$. For the second passband, the measured center frequency

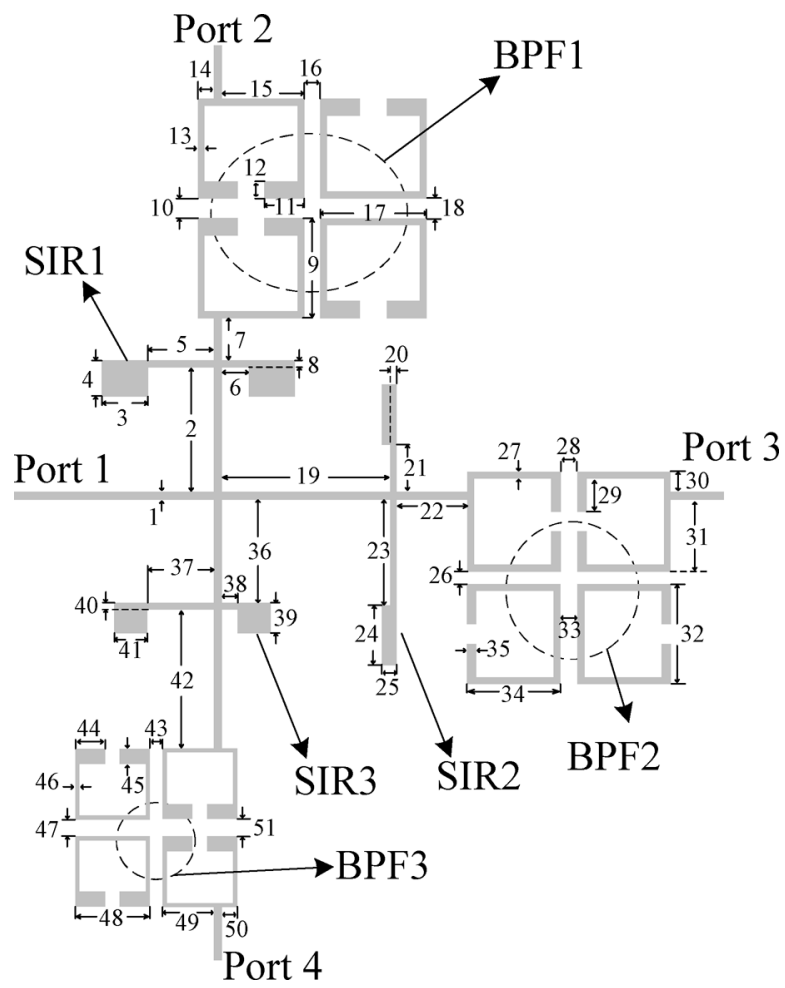

Fig. 5. Layout of the proposed microstrip triplexer.

TABLE I

DIMENSIONS (IN mm) OF EACH PART OF THE TRIPLEXER (FIG. 5)

\begin{tabular}{|c|c|c|c|c|c|c|c|c|c|c|}
\hline Part number & 1 & 2 & 3 & 4 & 5 & 6 & 7 & 8 & 9 & 10 \\
\hline Dimension & 1.2 & 22.2 & 6.9 & 5.35 & 11.92 & 2.08 & 3.4 & 0.5 & 15 & 0.94 \\
\hline Part number & 11 & 12 & 13 & 14 & 15 & 16 & 17 & 18 & 19 & 20 \\
\hline Dimension & 6 & 2.6 & 1 & 0.4 & 14.4 & 0.38 & 16 & 1.06 & 28.8 & 0.5 \\
\hline Part number & 21 & 22 & 23 & 24 & 25 & 26 & 27 & 28 & 29 & 30 \\
\hline Dimension & 7.58 & 10.6 & 16.42 & 9.5 & 1.2 & 0.4 & 1 & 1 & 5 & 1 \\
\hline & & & & & & & 27 & 30 & 30 & \\
\hline Dimension & 12.8 & 15 & 1.12 & 14 & 1.4 & 30.5 & 12 & 0.4 & 4.55 & 0.5 \\
\hline Part number & 41 & 42 & 43 & 44 & 45 & 46 & 47 & 48 & 49 & 50 \\
\hline Dimension & 4.95 & 46.4 & 0.43 & 4.5 & 2.25 & 0.6 & 1.02 & 11.2 & 9.7 & 0.3 \\
\hline Part number & 51 & & & & & & & & & \\
\hline Dimension & 1.12 & & & & & & & & & \\
\hline
\end{tabular}

$f_{02}$ is at $1.75 \mathrm{GHz}$, the minimum insertion loss is $3.57 \mathrm{~dB}$, and the $3-\mathrm{dB}$ bandwidth is $4.57 \%$. For the third passband, the measured center frequency $f_{03}$ is at $1.986 \mathrm{GHz}$, the minimum insertion loss is $3.64 \mathrm{~dB}$, and the $3-\mathrm{dB}$ bandwidth is $4.82 \%$. The isolation between the three passbands is better than $25 \mathrm{~dB}$, as shown in Fig. 7(b).

To illustrate the performance improvement due to matching circuits, a comparison of the triplexer responses with and without matching circuits is given in Fig. 8. The triplexer without matching circuits includes no SIRs and has only three $50-\Omega$ branch lines of lengths, such as $9 \mathrm{~mm}$, which are not 


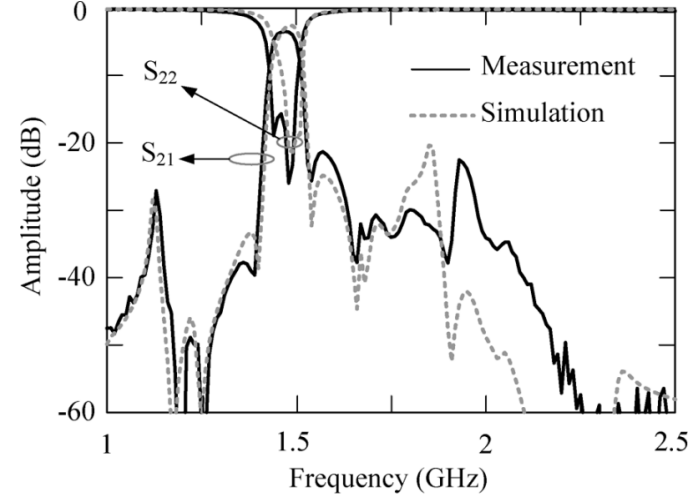

(a)

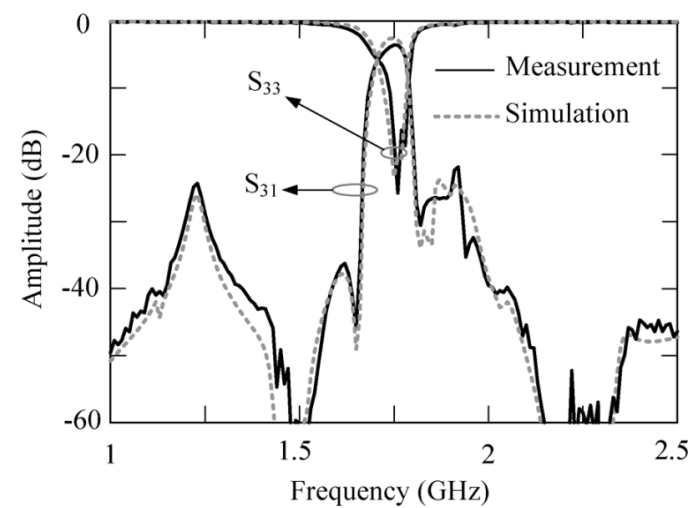

(b)

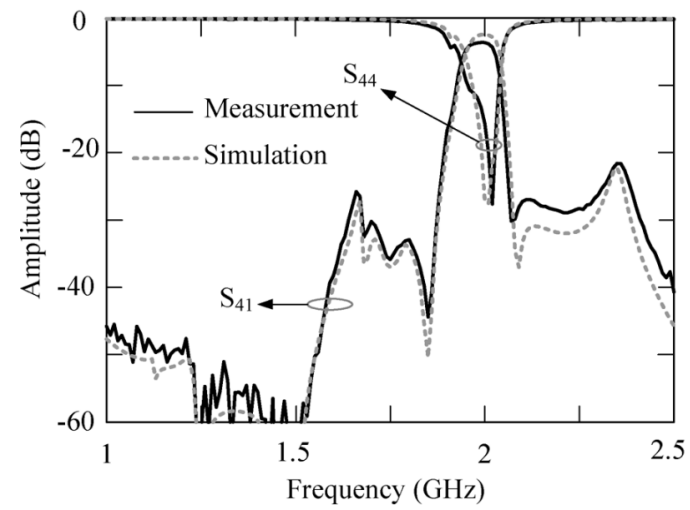

(c)

Fig. 6. Measured and simulated results of the proposed microstrip triplexer in Fig. 5. Frequency responses for: (a) the first passband, (b) the second passband, and (c) the third passband.

optimized. Obviously, an improvement in triplexer performance is observed due to the inclusion of SIRs in matching circuits.

The addition of matching elements unavoidably introduces additional insertion loss to the proposed triplexer circuit. On the average, the matching circuit adds $0.5 \mathrm{~dB}$ of insertion loss to the filter of each channel. Eventually, the three matching circuits introduce $0.9 \mathrm{~dB}$ of extra insertion loss to the resultant triplexer circuit due to the combination of three channels.

It is worth mentioning that unwanted spurious resonances would be introduced when the total length of any open stub is of $\lambda / 2$ in length. Fig. 9 explains the mechanism of inducing unwanted spurious resonances associated with channel 1, which consists of the bandpass filter BPF 1 together with its matching

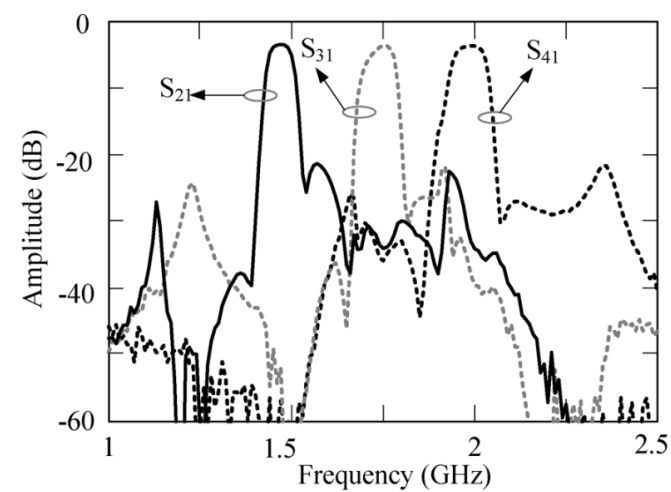

(a)

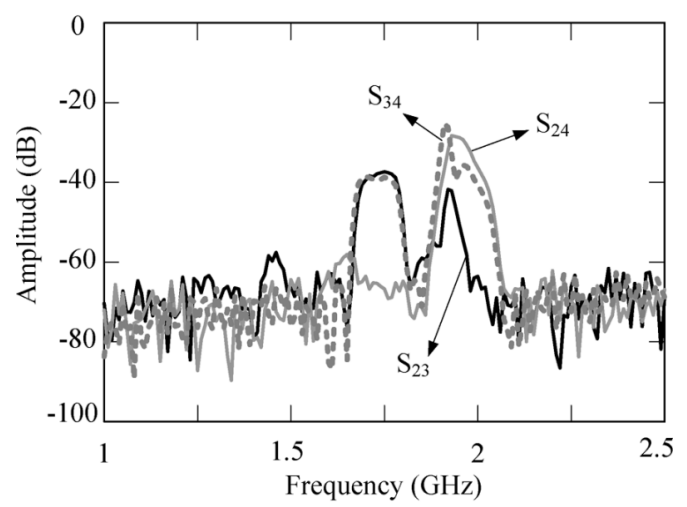

(b)

Fig. 7. Measured wideband responses of the microstrip in Fig. 5. (a) Insertion loss. (b) Isolation.

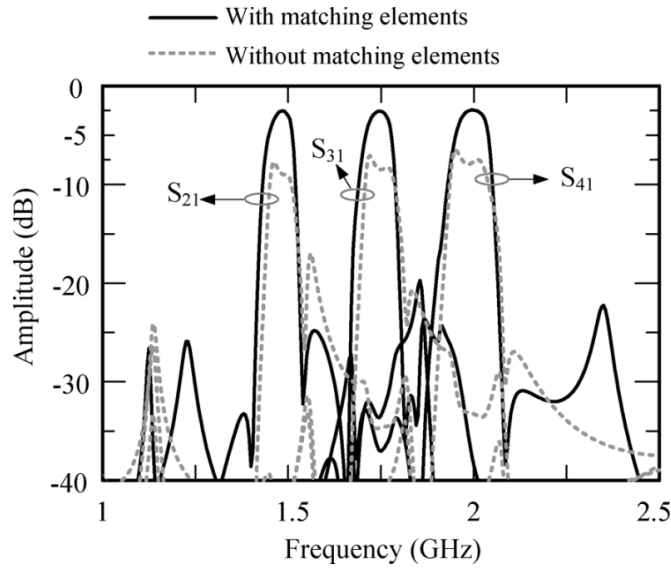

Fig. 8. Simulated responses of the triplexer with and without the matching elements.

element. Specifically, two spurious resonances are observed at 1.12 and $1.87 \mathrm{GHz}$, as shown in Fig. 9(b). These spurious resonances are generated when the open stubs denoted by $l_{h 1}$ and $l_{h 2}$ have their lengths approaching $\lambda / 2$ at 1.12 and $1.87 \mathrm{GHz}$, respectively. Intuitively, these spurious resonances may be improved by reducing the number of SIRs.

\section{Modified MATChing CiRCUITS FOR TRIPLEXERS}

To improve the spurious responses mentioned in Section III and to reduce the number of SIRs, modified matching circuits for the triplexer is also proposed. 


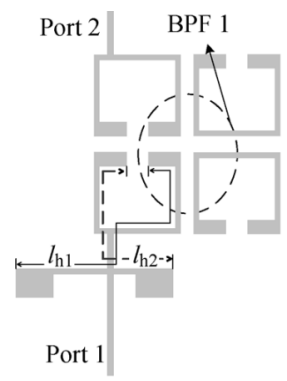

(a)

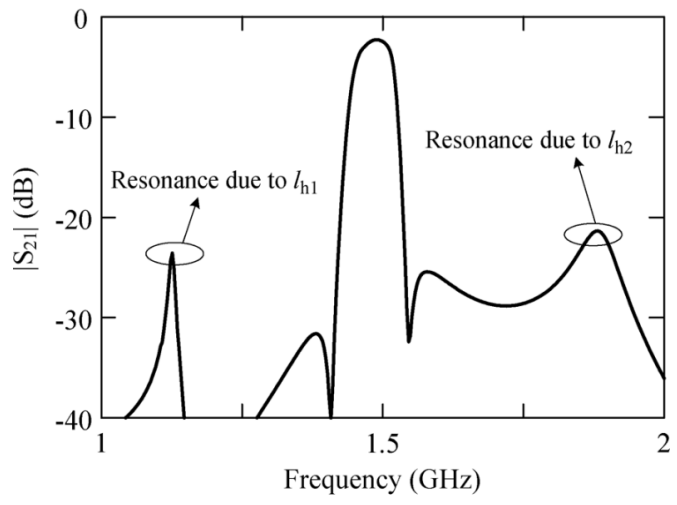

(b)

Fig. 9. Spurious resonances associated with the channel 1 of the triplexer in Fig. 5. (a) Layout. (b) Simulated responses.

Fig. 10(a) shows the architecture of the modified triplexer. For the branch lines, their characteristic impedances $Z_{0 k}, k=$ $1,2,3$, are preset to $50 \Omega$ as before, and their lengths $\left(l_{1}, y_{1}\right)$, $\left(l_{2}, y_{2}\right)$, and $y_{3}$ are properly chosen according to the criteria given below.

SIR1 is designed to resonate at $f_{01}$ so as to serve as a through pass at $f_{01}$ as before; therefore, the condition $Z_{\operatorname{in} 1}\left(f_{01}\right)=50 \Omega$ may be fulfilled. The tap position of SIR1 is also selected so that it may provide a short circuit again at $f_{02}\left[Z_{\mathrm{in} 1}^{\prime}\left(f_{02}\right)=0\right]$. The branch line is so designed that its length $l_{1}=\lambda / 4$ at $f_{02}$ and hence the open condition $Z_{\text {in1 }}\left(f_{02}\right)=\infty$ is met. Finally, the branch line $y_{1}$ is adjusted such that the open condition $Z_{\text {in1 } 1}\left(f_{03}\right)=\infty$ is satisfied. The design procedure for the first matching circuit is similar to the one given in Section III.

For the second and third matching circuits shown in Fig. 10(a), the branch-line length $y_{2}$ is chosen to satisfy the condition $Z_{\mathrm{in} 2}^{\prime}\left(f_{03}\right)=\infty$ and the length $y_{3}$ is selected to met the condition $Z_{\mathrm{in} 3}^{\prime}\left(f_{02}\right)=\infty$, respectively. Finally, the length $l_{2}$ is adjusted to fulfill the condition $Z_{\text {in } 2}\left(f_{01}\right)=\infty$. By adjusting the branch-line lengths according to the above-mentioned criteria, the required matching conditions may be satisfied again, and the design procedure is thus completed.

Note that the filters BPF2 and BPF3 are designed based on the reference impedance $50 \Omega$ at their input and output ports; therefore, the characteristic impedances of branch lines are better selected such that $Z_{02}=Z_{03}=50 \Omega$. If these characteristic impedances are not identical or not equal to $50 \Omega$, some reflections at the input ports of BPF2 and BPF3 may be produced.

The microstrip triplexer, based on the configuration in Fig. 10(a), which uses three fourth-order cross-coupled bandpass filters as in [18], is implemented with its layout shown in

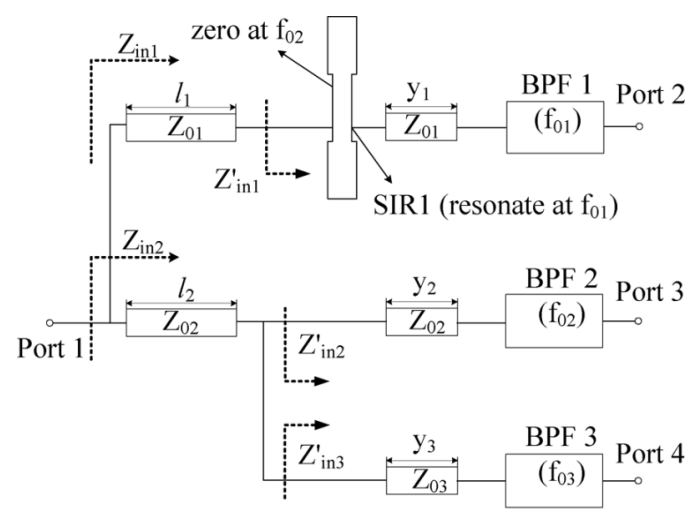

(a)

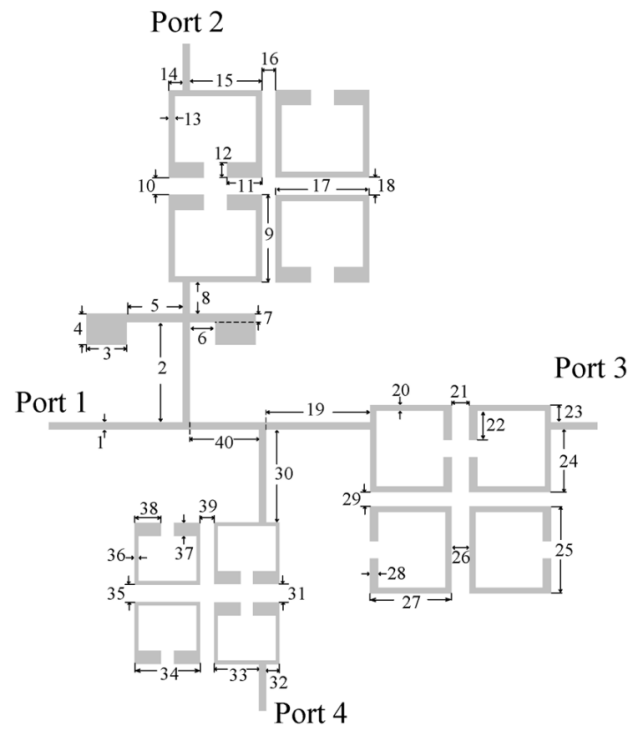

(b)

Fig. 10. (a) Structure and (b) layout of the modified microstrip triplexer.

TABLE II

Dimensions (IN Millimeters) OF EACH PART IN THE MODIFIED TRIPLEXER [SEE FIG. 10(b)]

\begin{tabular}{|c|c|c|c|c|c|c|c|c|c|c|}
\hline Part number & 1 & 2 & 3 & 4 & 5 & 6 & 7 & 8 & 9 & 10 \\
\hline Dimension & 1.2 & 22.2 & 6.9 & 5.35 & 11.62 & 2.38 & 0.5 & 3.4 & 15 & 0.94 \\
\hline & & & & & & & & & & \\
\hline rant & 11 & 12 & 15 & 14 & 15 & 10 & 17 & 10 & 19 & 20 \\
\hline Dimension & 6 & 2.6 & 1 & 0.4 & 14.4 & 0.38 & 16 & 1.06 & 25.9 & 1 \\
\hline Part number & 21 & 22 & 23 & 24 & 25 & 26 & 27 & 28 & 29 & 30 \\
\hline Dimension & 1 & 5 & 1 & 12.8 & 15 & 1.12 & 14 & 1.4 & 0.4 & 22.5 \\
\hline & & & & & & & & & & \\
\hline Part number & 31 & 32 & 33 & 34 & 35 & 36 & 37 & 38 & 39 & 40 \\
\hline Dimension & 11.2 & 0.3 & 9.7 & 11.2 & 1.02 & 0.6 & 2.25 & 4.5 & 0.43 & 11.9 \\
\hline
\end{tabular}

Fig. 10(b). The dimensions of each part in Fig. 10(b) are also given in Table II. The implemented triplexer has a dimension of $87.78 \mathrm{~mm} \times 103.12 \mathrm{~mm}$.

The measured and simulated results of the implemented modified triplexer Fig. 10(b) are shown in Figs. 11 and 12. For the first passband, the measured center frequency is at $1.483 \mathrm{GHz}$, the minimum insertion loss is $3.38 \mathrm{~dB}$, and the $3-\mathrm{dB}$ bandwidth 


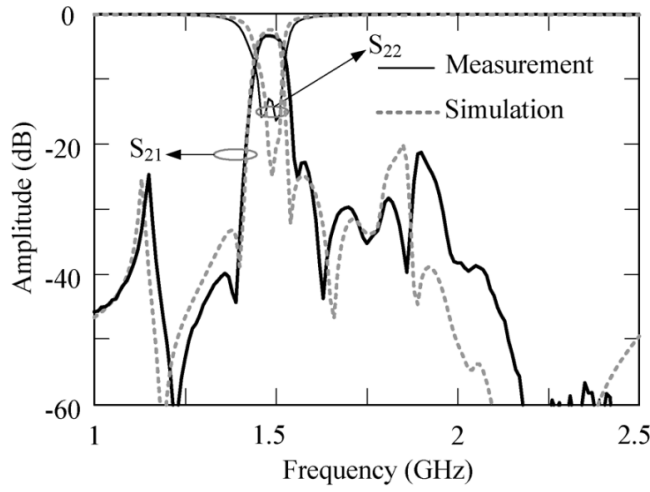

(a)

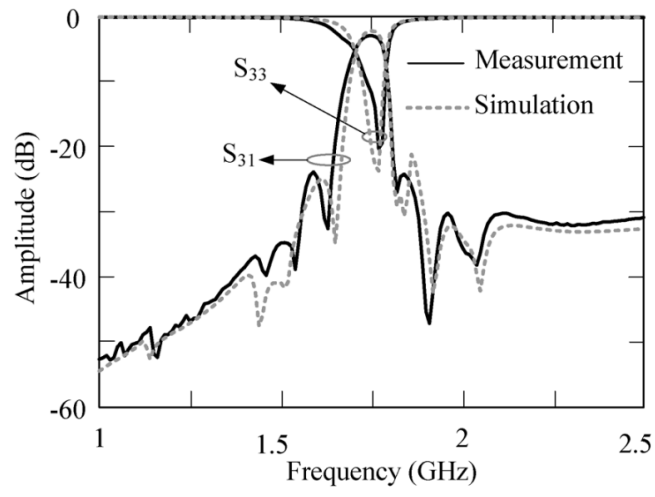

(b)

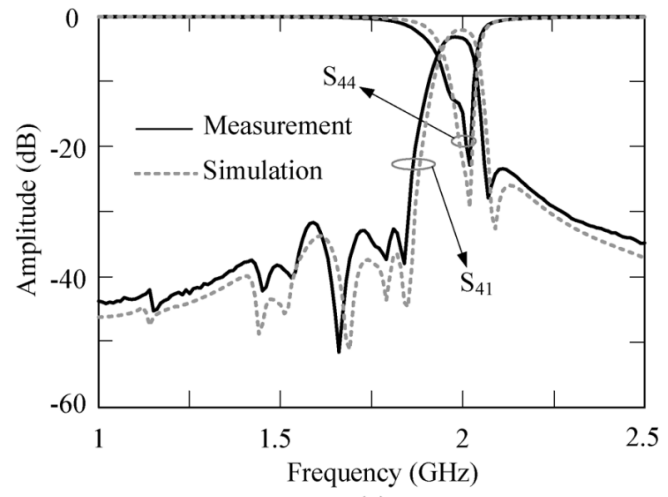

(c)

Fig. 11. Measured and simulated results of the modified microstrip triplexer in Fig. 10(b). Frequency responses for: (a) the first passband, (b) the second passband, and (c) the third passband.

is $5.35 \%$. For the second passband, the measured center frequency is at $1.75 \mathrm{GHz}$, the minimum insertion loss is $2.94 \mathrm{~dB}$, and the $3-\mathrm{dB}$ bandwidth is $5.6 \%$. For the third passband, the measured center frequency is at $1.984 \mathrm{GHz}$, the minimum insertion loss is $3.23 \mathrm{~dB}$, and the $3-\mathrm{dB}$ bandwidth is $5.04 \%$. The isolation between the three passbands is better than $32.58 \mathrm{~dB}$, as shown in Fig. 12(b).

For the output signals from ports 3 and 4 of the structure in Fig. 10(b), their spurious responses shown in Fig. 11(b) and (c) have been improved when compared with the ones shown in Fig. 6(b) and (c). This is due to the fact that the number of SIRs used in matching circuits is reduced, as shown in Fig. 10(b). Note that some spurious responses are also observed in Fig. 11(a) due to the use of the SIR in forming the matching circuit for port 2, as shown in Fig. 10(b).

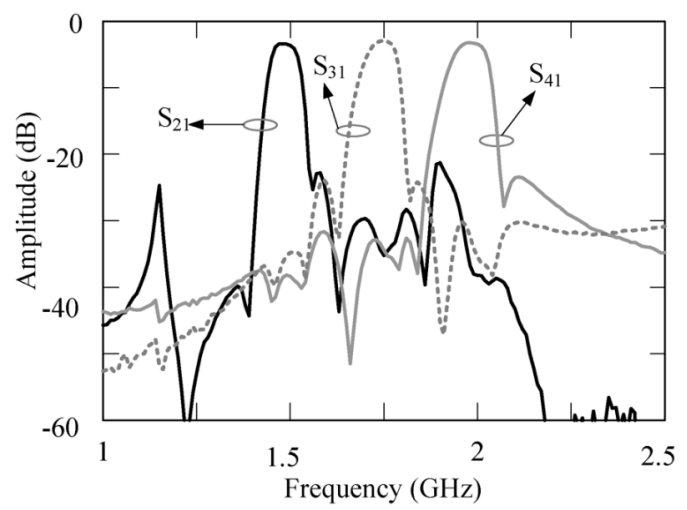

(a)

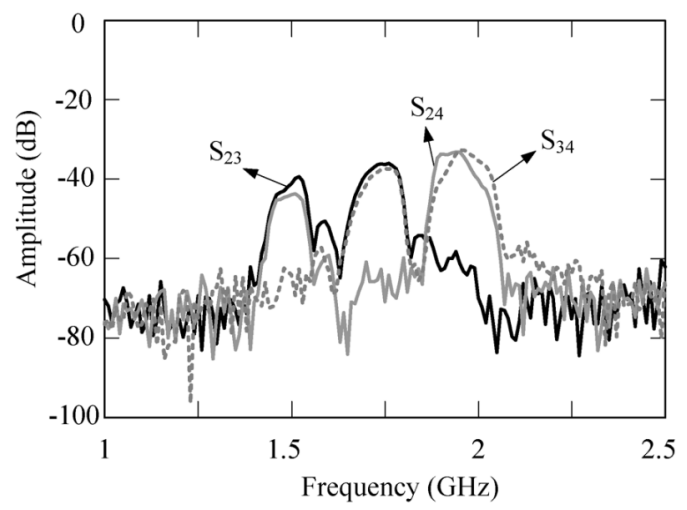

(b)

Fig. 12. Measured wideband responses of the triplexer in Fig. 10(b). (a) Insertion loss. (b) Isolation.

\section{CONCLUSION}

New matching circuits for microstrip triplexers have been proposed based on $\lambda / 2$ tapped-connected SIRs. The design procedure of the proposed matching circuits is simple and may be followed easily. In this study, two microstrip triplexer structures have been proposed. The first triplexer uses three tapped-connected SIRs to design the matching circuits for the triplexer. However, it has several unwanted spurious resonances in the stopband. In order to improve these spurious responses and to reduce the number of SIRs, the modified triplexer structure using only one tapped-connected SIR has been proposed. Both of the proposed triplexer structures are implemented and carefully examined.

\section{REFERENCES}

[1] D. M. Pozar, Microwave Engineering, 2nd ed. New York: Wiley, 1998, ch. 8

[2] A. F. Sheta, J. P. Coupez, G. Tanne, S. Toutain, and J. P. Blot, "Miniature microstrip stepped impedance resonator bandpass filters and diplexers for mobile communications," in IEEE MTT-S Int. Microw. Symp. Dig., Jun. 1996, pp. 607-610.

[3] C. M. Tsai, S. Y. Lee, C. C. Chuang, and C. C. Tsai, "A folded coupledline structure and its application to filter and diplexer design," in IEEE MTT-S Int. Microw. Symp. Dig., Jun. 2002, pp. 1927-1930.

[4] G. A. Lee, M. Megahed, and F. D. Flaviis, "Design of multilayer spiral inductor resonator filter and diplexer for system-in-a-package," in IEEE MTT-S Int. Microw. Symp. Dig., Jun. 2003, pp. 527-530.

[5] S. Srisathit, S. Patisang, R. Phromloungsri, S. Bunnjaweht, S. Kosulvit, and M. Chongcheawchamnan, "High isolation and compact size microstrip hairpin diplexer," IEEE Microw. Wireless Compon. Lett., vol. 15, no. 2, pp. 101-103, Feb. 2005. 
[6] R. R. Mansour, S. Ye, V. Dokas, B. Jolley, W.-C. Tang, and M. Kudsia, "Design considerations of superconductive input multiplexers for satellite applications," IEEE Trans. Microw. Theory Tech., vol. 44, no. 7, pp. 1213-1218, Jul. 1996.

[7] S. J. Fiedziuszko, J. A. Curtis, C. Holme, and R. S. Kwok, "Low loss multiplexers with planar dual-mode HTS resonators," IEEE Trans. Microw. Theory Tech., vol. 44, no. 7, pp. 1248-1257, Jul. 1996.

[8] T. Ohno, K. Wada, and O. Hashimoto, "A class of a planar triplexer by manipulating multiple attenuation poles," in Proc. 34th Eur. Microw. Conf., Oct. 2004, pp. 625-628.

[9] — "Design methodologies of planar duplexers and triplexers by manipulating attenuation poles," IEEE Trans. Microw. Theory Tech., vol. 53, no. 7, pp. 2088-2095, Jun. 2005.

[10] C. W. Tang and S. F. You, "Design methodologies of LTCC bandpass filters, diplexer, and triplexer with transmission zeros," IEEE Trans. Microw. Theory Tech., vol. 54, no. 2, pp. 717-723, Feb. 2006.

[11] M. I. Lai and S. K. Jeng, "A microstrip three-port and four-channel multiplexer for WLAN and UWB coexistence," IEEE Trans. Microw. Theory Tech., vol. 53, no. 10, pp. 3244-3250, Oct. 2005.

[12] J. S. Hong and M. J. Lancaster, "Theory and experiment of novel microstrip slow-wave open-loop resonator filters," IEEE Trans. Microw. Theory Tech., vol. 45, no. 12, pp. 2358-2365, Dec. 1997.

[13] S. Y. Lee and C. M. Tsai, "New cross-coupled filter design using improved hairpin resonators," IEEE Trans. Microw. Theory Tech., vol. 48, no. 12, pp. 2482-2490, Dec. 2000.

[14] C. M. Tsai, S. Y. Lee, and C. C. Tsai, "Performance of a planar filter using a $0^{\circ}$ feed strucure," IEEE Trans. Microw. Theory Tech., vol. 50, no. 10, pp. 2362-2367, Oct. 2002.

[15] P. H. Deng, S. C. Lin, Y. S. Lin, C. H. Wang, and C. H. Chen, "Microstrip bandpass filters with dissimilar resonators for suppression of spurious responses," in Proc. 35th Eur. Microw. Conf., Paris, France, Oct. 2005, pp. 1263-1266.

[16] S. C. Lin, P. H. Deng, Y. S. Lin, C. H. Wang, and C. H. Chen, "Wide-stopband microstrip bandpass filters using dissimilar quarter-wavelength stepped-impedance resonators," IEEE Trans. Microw. Theory Tech., vol. 54, no. 3, pp. 1011-1018, Mar. 2006.

[17] M. Sagawa, M. Makimoto, and S. Yamashita, "Geometrical structures and fundamental characteristics of microwave stepped-impedance resonators," IEEE Trans. Microw. Theory Tech., vol. 45, no. 7, pp. 1078-1085, Jul. 1997.

[18] J. S. Hong and M. J. Lancaster, "Couplings of microstrip square open-loop resonators for cross-coupled planar microwave filters," IEEE Trans. Microw. Theory Tech., vol. 44, no. 11, pp. 2099-2109, Nov. 1996.

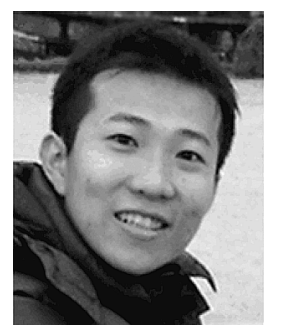

Pu-Hua Deng was born in Kaohsiung, Taiwan, R.O.C., in 1978. He received the B.S. degree in electrical engineering from National Sun Yet-Sen University, Kaohsiung, Taiwan, R.O.C., in 2002, the M.S.E.E. degree from National Taiwan University, Taipei, Taiwan, R.O.C., in 2004, and the Ph.D. degree from National Taiwan University, Taipei, Taiwan, R.O.C. in 2006.

His research interests include the design and analysis of microwave filter circuits.

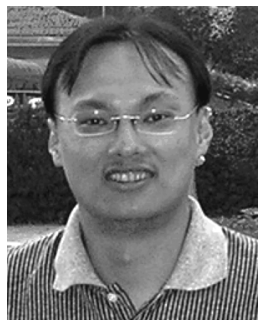

Ming-Iu Lai (S'04) was born in Kaohsiung, Taiwan, R.O.C., in 1976. He received the B.S.E.E. degree from National Taiwan University of Science and Technology, Taipei, Taiwan, R.O.C., in 1998, the M.S.E.E. degree from National Taiwan University, Taipei, Taiwan, R.O.C., in 2000, and is currently working toward the Ph.D. degree at National Taiwan University.

From 2001 to 2002, he was with the ZyXEL Communication Corporation, Hsinchu, Taiwan, R.O.C., where he involved with signal integrity (SI) and electromagnetic interference (EMI) analyses. From 2002 to 2004, he was with the SynComm Communication Corporation, Hsinchu, Taiwan, R.O.C. He then joined the Applied Electromagnetic Research Laboratory, Microelectronics and Information System Research Center, National Chiao Tung University, Hsinchu, Taiwan, R.O.C., where he was involved with the designs of beam-steering antennas and high-frequency flip-chip packages. His current research interest includes array antennas and multiple-input and multiple-output antenna designs.

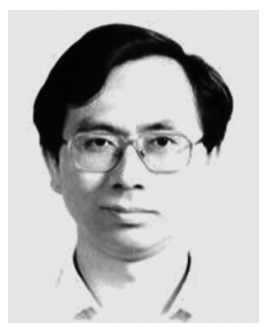

Shyh-Kang Jeng (M'86-SM'98) received the B.S.E.E. and Ph.D. degrees from National Taiwan University, Taipei, Taiwan, R.O.C., in 1979 and 1983, respectively.

In 1981, he joined the faculty of the Department of Electrical Engineering, National Taiwan University, where he is currently a Professor. From 1985 to 1993 , he was with the University of Illinois, Urbana-Champaign, as a Visiting Research Associate Professor and a Visiting Research Professor. For six months in 1999, he was with the Center for Computer Research in Music and Acoustics, Stanford University, Stanford, CA. His research interest includes numerical electromagnetics, ultra-wideband wireless systems, music signal processing, music information retrieval, intelligent agent applications, and electromagnetic scattering analysis.

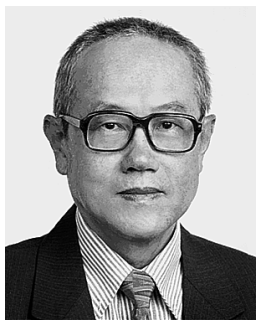

Chun Hsiung Chen (SM'88-F'96) was born in Taipei, Taiwan, R.O.C., on March 7, 1937. He received the B.S.E.E. degree in electrical engineering from National Taiwan University, Taipei, Taiwan, R.O.C., in 1960, the M.S.E.E. degree from National Chiao Tung University, Hsinchu, Taiwan, R.O.C., in 1962, and the Ph.D. degree in electrical engineering from National Taiwan University, in 1972.

In 1963, he joined the Faculty of the Department of Electrical Engineering, National Taiwan University, where he is currently a Professor. From August 1982 to July 1985, he was Chairman of the Department of Electrical Engineering, National Taiwan University. From August 1992 to July 1996, he was the Director of the University Computer Center, National Taiwan University. In 1974, he was a Visiting Scholar with the Department of Electrical Engineering and Computer Sciences, University of California at Berkeley. From August 1986 to July 1987, he was a Visiting Professor with the Department of Electrical Engineering, University of Houston, Houston, TX. In 1989, 1990, and 1994, he visited the Microwave Department, Technical University of Munich, Munich, Germany, the Laboratoire d'Optique Electromagnetique, Faculte des Sciences et Techniques de Saint-Jerome, Universite d'Aix-Marseille III, Marseille, France, and the Department of Electrical Engineering, Michigan State University, East Lansing, respectively. His areas of interest include microwave circuits and computational electromagnetics. 